\title{
The Effect of Stress and Depression on Gastrointestinal Diseases
}

TO THE EDITOR: In a recent issue of the Journal of Neurogastroenterology and Motility, we were very interested in the article by Lee et $\mathrm{al}^{1}$ entitled "The Effect of emotional stress and depression on the prevalence of digestive diseases" in which the investigators reported that stress and depression are related to various digestive diseases and may be predisposing factors for functional dyspepsia and irritable bowel syndrome, and that depression may be a predisposing factor for gastric cancer. This study is well designed but we would like to comment on some of the factors that can affect depression and stress as these may shed new light on the author's interpretations.

Large-scale studies that aim to determine psychological processes such as depression and stress levels in psychosomatic diseases such as gastrointestinal diseases should meticulously consider the risk factors that affect these processes. At the same time, the inclusion and exclusion criteria should be well structured. For example, the main factors that affect stress and depression levels are psychotropic medication and regular exercise. We know that both psychotropic medication ${ }^{2}$ and regular exercise ${ }^{3}$ decrease levels of depression and stress. The study by Lee et $\mathrm{al}^{1}$ did not specify whether or not participitants used psychotropic medication or exercised regularly, nor did it specify if these factors were used as exclusion or inclusion criteria.

In addition, most women begin to experience emotional changes before and during menstruation period which are known as premenstrual syndrome (PMS). ${ }^{4}$ Symptoms of PMS include affective, physical, cognitive, and behavioral changes. Affective symptoms include irritability (ie, a cardinal symptom), mood swings, anxiety, and depression. ${ }^{5-6}$ These psychological symptoms do not only occur during PMS but can also be seen during and after menopause. Upon entering menopause, women may experience a wide range of feelings, from anxiety and discomfort to release and relief. ${ }^{7-8}$ Thus, stress and depression levels show fluctuations during the menstrual cycle and menopausal period.
In this study, the authors did not state the menstrual status of female individuals which could cause unnaturally lower or higher stress and depression levels.

We think that it is highly important to examine these issues in order to reliably interpret the study results. Clarifying these 2 concerns will provide a clearer picture when interpreting stress and depression levels among participants.

Cemil Celik, Barbaros Ozdemir, and Taner Oznur Department of Psychiatry, Gulhane Military Medical Faculty, Ankara, Turkey

1. Lee SP, Sung IK, Kim JH, Lee SY, Park HS, Shim CS. The effect of emotional stress and depression on the prevalence of digestive diseases. J Neurogastroenterol Motil 2015;21:273-282.

2. Castle MZ, Silk DB, Libby GW. Review article: rhe rationale for antidepressant therapy in functional gastrointestinal disorders. Aliment Pharmacol Ther 2004;19:969-979.

3. Byrne A, Byrne DG. The effect of exercise on depression, anxiety and other mood states: a review. J Psychosom Res 1993;37:565-574.

4. Yonkers KA, O'Brien PM, Eriksson E. Premenstrual syndrome. Lancet 2008;371:1200-1210.

5. Rapkin AJ, Mikacich JA. Premenstrual dysphoric disorder and severe premenstrual syndrome in adolescents. Paediatr Drugs 2013;15: 191-202.

6. Gao X, Sun P, Qiao M, Wei S, Xue L, Zhang H. Shu-Yu capsule, a traditional Chinese medicine formulation, attenuates premenstrual syndrome depression induced by chronic stress constraint. Mol Med Rep 2014;10:2942-2948.

7. Bromberger JT, Matthews KA, Schott LL, et al. Depressive symptoms during the menopausal transition: the Study of Women's Health Across the Nation (SWAN). J Affect Disord 2007;103: 267-272.

8. Cohen LS, Soares CN, Vitonis AF, Otto MW, Harlow BL. Risk for new onset of depression during the menopausal transition: the Harvard study of moods and cycles. Arch Gen Psychiatry 2006;63: 385-390.

\section{Conflicts of interest: None.}

